\title{
Shape reconstruction from point clouds using closed form solution of a fourth-order partial differential equation
}

\author{
Zaiping Zhu ${ }^{1}$, Ehtzaz Chaudhry ${ }^{1}$, Shuangbu Wang ${ }^{1}, Y$ Xia $^{1}$, Andres Iglesias ${ }^{2}$, \\ Lihua You ${ }^{1}$, and Jian Jun Zhang ${ }^{1}$ \\ ${ }^{1}$ The National Center for Computer Animation, Bournemouth University, UK \\ 2 Department of Applied Mathematics and Computational Sciences, University of Cantabria, \\ Cantabria 39005, Spain
}

\begin{abstract}
Partial differential equation (PDE) based geometric modelling has a number of advantages such as fewer design variables, avoidance of stitching adjacent patches together to achieve required continuities, and physics-based nature. Although a lot of papers have investigated PDE-based shape creation, shape manipulation, surface blending and volume blending as well as surface reconstruction using implicit PDE surfaces, there is little work of investigating PDEbased shape reconstruction using explicit PDE surfaces, specially satisfying the constraints on four boundaries of a PDE surface patch. In this paper, we propose a new method of using an accurate closed form solution to a fourth-order partial differential equation to reconstruct 3D surfaces from point clouds. It includes selecting a fourth-order partial differential equation, obtaining the closed form solutions of the equation, investigating the errors of using one of the obtained closed form solutions to reconstruct PDE surfaces from different number of $3 \mathrm{D}$ points.
\end{abstract}

Keywords: Shape reconstruction, fourth-order partial differential equation, closed form solutions, error analysis.

\section{Introduction}

Shape reconstruction has a lot of applications in many fields. Various surface reconstruction methods have been developed. These methods include polygon-based, implicit surface-based, and parametric surface-based. In addition, soft computing is also introduced into parametric surfaces to improve shape reconstruction from point clouds.

Shape reconstruction uses polygon meshes, implicit surfaces, and existing parametric surfaces such as Bézier, B-spline, and NURBS surfaces has some weaknesses. They include bid data, heavy geometry processing, high data storage cost, and slow data transmission over computer networks. How to address these weaknesses is an unsolved topic.

In contrast, PDE-based shape reconstruction has the following advantages. First, a single PDE surface patch can describe a complicated shape leading to smaller data than NURBS, polygon and subdivision modelling techniques. Second, adjacent PDE surface patches naturally maintain position, tangent, or higher continuities and no manual operations are required to stitch different PDE patches together. Third, any irregular 
boundaries can be quickly specified by drawing a closed curve on 3D models, different sculpting forces can be applied to achieve the expected shape, and global shape manipulations can be easily obtained through shape control parameters etc., leading to more efficient shape manipulations.

However, a main difficulty for PDE-based shape manipulation is how to solve partial differential equations. Due to this difficulty, most studies investigated implicit PDEbased shape reconstruction which involves numerically solving partial differential equations. Although some research studies investigated explicit PDE-based shape reconstruction by interpolating four curves or satisfying the constraints on two opposite boundaries of a PDE patch, few studies presented closed form solutions of partial differential equations for 4-sided PDE patches. In this paper, we will propose a mathematical model, derive its closed form solutions, and use one of the closed form solutions to achieve shape reconstruction from point clouds.

\section{$2 \quad$ Related Work}

There are a lot of work of investigating shape reconstruction from point clouds. A comprehensive literature survey has been made in [1,2]. Among these shape reconstruction methods, PDE-based shape reconstruction has also been investigated. The existing shape reconstruction methods can be divided into polygon-based, implicit surfacebased, explicit surface-based, and soft computing and parametric surface-based. Shape reconstruction using explicit surfaces from solutions to partial differential equations was summarized in [3]. In this section, we briefly review some work on shape reconstruction from point clouds.

Polygon-based shape reconstruction is most popular. Many of them are based on the Delaunay triangulation. For each initial border edge in triangulated reconstruction, Boissonnant estimated a tangential plane and took a vertex of a surface triangle to be the sample point which maximizes the angle between the vertex and the $k$-nearest neighbors projected to the tangential plane [4], Hoppe et al. used $k$-nearest neighbors to find a tangential plan of every sample point and the marching cubes algorithm and the signed distance of the sample point closest to the tangential plan to reconstruct 3D surfaces [5]. Oblonšek and Guid presented a procedure to triangulate the input scattered point set, extract features, and fair the triangular mesh to achieve surface reconstruction [6]. Bernardini et al. proposed a ball-pivoting algorithm to interpolate a given point cloud to reconstruct a triangle mesh where a triangle is formed with three points when a ball touches them [7]. Gopi et al. projected the neighborhood of each of sample points on a tangential plan, derived the 2D Delaunay triangulation on the tangential plan, and mapped the result back to the 3D space, and reconstructed a 3D surface from the mapping [8]. Lee et al. reconstructed a 3D surface through repeated subdivision, displacement, and resampling of a control mesh model [9]. Jeong and Kim constructed a coarse base mesh from a bounding cube containing the input point cloud, and successively subdivide, smooth, and project it to the input point cloud to obtain shape reconstruction [10]. Not fitting dense smooth polygonal surfaces, Nan and Wonka used simple polygonal surfaces for reconstruction of piecewise planar objects [11]. 
Implicit surface-based shape reconstruction has also been extensively investigated. Duan et al. proposed a PDE-based deformable surface to reconstruct 3D models from volumetric data, unorganized point clouds and multi-view 2D images [12]. Linz et al. developed new formulations and fast algorithms to conduct implicit partial differential equation-based surface reconstruction [13]. Franchini et al. used the numerical technique based on an efficient semi-implicit scheme and finite volume space discretization to solve a time-dependent partial differential equation for implicit shape reconstruction [14]. Pana and Skala introduced an energy functional to combine flux-based data-fit measures and proposed a regulation term and a continuous global optimization method to carry out surface reconstruction from an oriented point cloud [15]. Using an implicit and continuous representation of reconstructed surfaces and optimizing a regularized fitting energy, Liu et al. developed a level-set based surface reconstruction method to process point clouds captured by a surface photogrammetry system [16]. He et al. presented two fast algorithms, one uses the semi-Implicit method and the other is based on the augmented Lagrangian method, to improve computational efficiency of surface reconstruction from point clouds [17].

Some researchers investigated explicit surface-based shape reconstruction. In order to solve the heavy computational cost of finite element methods or finite difference methods in solving the elliptic partial differential equation for 3D shape reconstruction, $\mathrm{Li}$ ang Hero developed a fast spectral method to improve the performance [18]. Ugail and Kirmani proposed an explicit partial differential equation-based reconstruction method which interpolates four curves parametrized in terms of the parametric variable $v$ to obtain a reconstructed surface [19]. This method was also used to obtain reconstruction of 3D human facial images [20]. Rodrigues et al. obtained an analytical solution of a Laplace equation and used it in 3D data compression and reconstruction [21]. Sheng et al. integrated a point cloud update algorithm, a rapid iterative closest point algorithm, and an improved Poisson surface reconstruction algorithm together to improve the efficiency of surface reconstruction [22].

Soft computing has also been introduced into parametric surfaces to optimize shape reconstruction. Iglesias et al. introduced function networks into B-spline surfaces to solve the problem of shape reconstruction [23]. Gálvez and Iglesias integrated an iterative two-step genetic-algorithm and polynomial B-spline surfaces for efficient surface reconstruction from point clouds [24]. They proposed a particle swarm optimization approach to reconstruct non-uniform rational B-spline surfaces from 3D point clouds [25].

Since few research studies investigated shape reconstruction using explicit PDE surfaces, we will tackle this issue in this paper. The PDE mathematical model and its closed form solutions will be investigated in Section 3. The proposed PDE-based shape reconstruction and error analysis are examined in Section 4. Finally, the conclusion is drawn and some future research directions are discussed in section 5.

\section{$3 \quad$ Mathematical model and closed form solution}

Partial differential equation-based shape reconstruction can be roughly divided into two categories: one uses implicit solutions of partial differential equations and the other 
uses explicit solutions of partial differential equations. Shape reconstruction using implicit solutions of partial differential equations involves a lot of numerical calculations, causing slow shape reconstruction which is not suitable for many applications requiring real-time performance. In contrast, shape reconstruction using explicit solutions of partial differential equation is based on accurate analytical or approximate analytical solutions of partial differential equations, which involves fewer calculations and is more efficient than shape construction using implicit solutions of partial differential equations.

However, a main problem for shape reconstruction using explicit solutions of partial differential equations is how to obtain accurate analytical or approximate analytical solutions of partial differential equations. Since solving partial differential equations analytically is not an easy task, the current explicit solutions of partial differential equations used for PDE-based geometric modelling and shape reconstruction mainly deal with two boundaries of a PDE surface patch, i. e., accurately satisfy partial differential equations and continuity constraints at two opposite boundaries of a PDE surface patch. How to obtain accurate analytical solutions of partial differentia equations which exactly satisfy partial differential equations and continuity constraints on four boundaries of a PDE surface patch is an important topic.

A vector-valued partial differential equation used to describe a 3D surface patch involves two parametric variables $u$ and $v$. The four boundaries of the 3D surface patch are defined by $u=0, u=1, v=0$, and $v=1$. In order to satisfy positional continuities, four unknowns should be included in a closed form solution to a vector-valued partial differential equation to satisfy four positional functions, i. e. boundary curves at the four boundaries of a 3D surface patch. Similarly, in order to satisfy up to tangential continuities, eight unknowns should be involved in a closed form solution of a vectorvalued partial differential equation to satisfy four positional functions and four tangential functions at the four boundaries of a 3D surface patch. From the theory of partial differential equation, the closed form solution to a second-order partial differential equation of parametric variables $u$ and $v$ has four unknowns, and the closed form solution to a fourth-order partial differential equation has eight unknowns.

Up to tangential continuities is most popularly used to create smooth 3D models. Taking all of these factors and a closed form solution into account, we propose to use the following vector-valued fourth-order partial differential equation for shape reconstruction

$$
a_{1} \frac{\partial^{4} \mathbf{X}(u, v)}{\partial u^{4}}+a_{2} \frac{\partial^{4} \mathbf{X}(u, v)}{\partial v^{4}}=\mathbf{F}(u, v)
$$

where $\boldsymbol{a}_{1}$ and $\boldsymbol{a}_{2}$ are called vector-value shape control parameters, which can be used to change the shape of a PDE surface without changing boundary continuities and each of which has three components $a_{i x}, a_{i y}$, and $a_{i z}(i=1,2)$ with $\boldsymbol{a}_{1}=$ $\left[\begin{array}{lll}a_{1 x} & a_{1 y} & a_{1 z}\end{array}\right]^{T}$ and $\boldsymbol{a}_{2}=\left[\begin{array}{lll}a_{2 x} & a_{2 y} & a_{2 z}\end{array}\right]^{T}, u$ and $v$ are two parametric variables defined by $0 \leq u \leq 1$ and $0 \leq v \leq 1, \mathbf{X}(u, v)$ is a vector-valued position function used to define a PDE surface patch, which has three components $x(u, v), y(u, v)$, and $z(u, v)$ with $\mathbf{X}(u, v)=\left[\begin{array}{llll}x(u, v) & y(u, v) & z(u, v)\end{array}\right]^{T}$, and $\mathbf{F}(u, v)$ is a vector-valued sculpting function, which also has three components $f_{x}(u, v) f_{y}(u, v)$ and $f_{z}(u, v)$ with $\mathbf{F}(u, v)=\left[\begin{array}{lll}f_{x}(u, v) & f_{y}(u, v) & f_{z}(u, v)\end{array}\right]^{T}$. The components of each of the two 
vector-valued shape control parameters cab be taken to be the same, i. e, $a_{i x}=a_{i y}=a_{i z}$ $(i=1,2)$ or different, i. e., $a_{i x} \neq a_{i y} \neq a_{i z}(i=1,2)$.

In order to simplify mathematical notations, we define the following mathematical operations in this paper

$$
\begin{aligned}
\boldsymbol{a}_{1} \boldsymbol{a}_{2} & =\left[\begin{array}{lll}
a_{1 x} a_{2 x} & a_{1 y} a_{2 y} & a_{1 z} a_{2 z}
\end{array}\right]^{T} \\
\frac{\boldsymbol{a}_{1}}{\boldsymbol{a}_{2}} & =\left[\begin{array}{lll}
\frac{a_{1 x}}{a_{2 x}} & \frac{a_{1 y}}{a_{2 y}} & \frac{a_{1 z}}{a_{2 z}}
\end{array}\right]^{T} \\
e^{\boldsymbol{a}_{1}} & =\left[\begin{array}{lll}
e^{a_{1 x}} & e^{a_{1 y}} & e^{a_{1 z}}
\end{array}\right]^{T} \\
n & \sqrt[n]{\frac{\boldsymbol{a}_{1}}{\boldsymbol{a}_{2}}}=\left[\begin{array}{lll}
\sqrt[n]{\frac{a_{1 x}}{a_{2 x}}} & \sqrt[n]{\frac{a_{1 y}}{a_{2 y}}} & \sqrt[n]{\frac{a_{1 z}}{a_{2 z}}}
\end{array}\right]^{T} \\
\cos \boldsymbol{a}_{1} & =\left[\begin{array}{lll}
\cos a_{1 x} & \cos a_{1 y} & \cos a_{1 z}
\end{array}\right]^{T} \\
\sin \boldsymbol{a}_{1} & =\left[\begin{array}{lll}
\sin a_{1 x} & \sin a_{1 y} & \sin a_{1 z}
\end{array}\right]^{T}
\end{aligned}
$$

In this paper, we look for closed form solutions of the homogeneous form of the partial differential equation (1) and use one of them for shape reconstruction from point clouds. In the extended version of this paper, we will investigate the particular solution of the partial differential equation (1) and use it to develop a more powerful shape reconstruction tool.

We use the method of separation of variables to solve the homogeneous form of the vector-valued partial differential equation (1) and obtain its four closed form solutions. The details of solving the homogeneous form of the vector-valued partial differential equation (1) will be given in the extended version of this paper. In what follows, we use one closed form solution among the four obtained closed form solutions to demonstrate shape reconstruction using the closed form solutions of the vector-valued fourthorder partial differential equation (1) and investigate the errors of shape reconstruction. The closed form solution to be used can be written in the following form

$$
\mathbf{X}(u, v)=\sum_{j=1}^{16} \boldsymbol{d}_{j} \boldsymbol{f}_{j}(u, v)
$$

where

$$
\begin{gathered}
\boldsymbol{f}_{1}(u, v)=e^{\boldsymbol{q}_{2} u} e^{\boldsymbol{q}_{4} v} \cos \boldsymbol{q}_{2} u \cos \boldsymbol{q}_{4} v \\
\boldsymbol{f}_{2}(u, v)=e^{\boldsymbol{q}_{2} u} e^{\boldsymbol{q}_{4} v} \cos \boldsymbol{q}_{2} u \sin \boldsymbol{q}_{4} v \\
\boldsymbol{f}_{3}(u, v)=e^{\boldsymbol{q}_{2} u} e^{\boldsymbol{q}_{4} v} \sin \boldsymbol{q}_{2} u \cos \boldsymbol{q}_{4} v \\
\boldsymbol{f}_{4}(u, v)=e^{\boldsymbol{q}_{2} u} e^{\boldsymbol{q}_{4} v} \sin \boldsymbol{q}_{2} u \sin \boldsymbol{q}_{4} v \\
\boldsymbol{f}_{5}(u, v)=e^{\boldsymbol{q}_{2} u} e^{-\boldsymbol{q}_{4} v} \cos \boldsymbol{q}_{2} u \cos \boldsymbol{q}_{4} v \\
\boldsymbol{f}_{6}(u, v)=e^{\boldsymbol{q}_{2} u} e^{-\boldsymbol{q}_{4} v} \cos \boldsymbol{q}_{2} u \sin \boldsymbol{q}_{4} v \\
\boldsymbol{f}_{7}(u, v)=e^{\boldsymbol{q}_{2} u} e^{-\boldsymbol{q}_{4} v} \sin \boldsymbol{q}_{2} u \cos \boldsymbol{q}_{4} v \\
\boldsymbol{f}_{8}(u, v)=e^{\boldsymbol{q}_{2} u} e^{-\boldsymbol{q}_{4} v} \sin \boldsymbol{q}_{2} u \sin \boldsymbol{q}_{4} v \\
\boldsymbol{f}_{9}(u, v)=e^{-\boldsymbol{q}_{2} u} e^{\boldsymbol{q}_{4} v} \cos \boldsymbol{q}_{2} u \cos \boldsymbol{q}_{4} v \\
\boldsymbol{f}_{10}(u, v)=e^{-\boldsymbol{q}_{2} u} e^{\boldsymbol{q}_{4} v} \cos \boldsymbol{q}_{2} u \sin \boldsymbol{q}_{4} v \\
\boldsymbol{f}_{11}(u, v)=e^{-\boldsymbol{q}_{2} u} e^{\boldsymbol{q}_{4} v} \sin \boldsymbol{q}_{2} u \cos \boldsymbol{q}_{4} v \\
\boldsymbol{f}_{12}(u, v)=e^{-\boldsymbol{q}_{2} u} e^{\boldsymbol{q}_{4} v} \sin \boldsymbol{q}_{2} u \sin \boldsymbol{q}_{4} v \\
\boldsymbol{f}_{13}(u, v)=e^{-\boldsymbol{q}_{2} u} e^{-\boldsymbol{q}_{4} v} \cos \boldsymbol{q}_{2} u \cos \boldsymbol{q}_{4} v \\
\boldsymbol{f}_{14}(u, v)=e^{-\boldsymbol{q}_{2} u} e^{-\boldsymbol{q}_{4} v} \cos \boldsymbol{q}_{2} u \sin \boldsymbol{q}_{4} v \\
\boldsymbol{f}_{15}(u, v)=e^{-\boldsymbol{q}_{2} u} e^{-\boldsymbol{q}_{4} v} \sin \boldsymbol{q}_{2} u \cos \boldsymbol{q}_{4} v \\
\boldsymbol{f}_{16}(u, v)=e^{-\boldsymbol{q}_{2} u} e^{-\boldsymbol{q}_{4} v} \sin \boldsymbol{q}_{2} u \sin \boldsymbol{q}_{4} v
\end{gathered}
$$


and

$$
\begin{gathered}
\boldsymbol{q}_{2}=\frac{\sqrt{2}}{2} \sqrt[4]{\mid \frac{\boldsymbol{c}_{0}}{\boldsymbol{a}_{1} \mid}} \\
\boldsymbol{q}_{4}=\frac{\sqrt{2}}{2} \sqrt[4]{\left|\frac{\boldsymbol{c}_{0}}{\boldsymbol{a}_{2}}\right|}
\end{gathered}
$$

where $\boldsymbol{d}_{j}(j=1,2,3, \cdots, 16)$ are the vector-valued unknowns, and $\boldsymbol{c}_{0}$ is a vector-valued constant.

$\mathbf{X}(u, v)$ in Eq. (3) defines a PDE surface patch. In the following section, we will discuss how to use it to achieve shape reconstruction for point clouds.

\section{Shape reconstruction and error analysis}

Shape reconstruction from point clouds is to find the 16 vector-valued unknowns $\boldsymbol{d}_{j}$ $(j=1,2,3, \cdots, 16)$ which make the PDE surface patch $\mathbf{X}(u, v)$ best fit the points in the region to be reconstructed. For any unorganized point clouds, we can find the points close to each of a set of planes, which define a curve close to the plane. From these curves, we can obtain the values of the parametric variable $u$ for these curves. From the points on each of the curves, we can obtain the values of the parametric variable $v$ for all the points on the curve. For the points not on these curves, we can use the geometric relationships of their positions relative to the points on the curves to obtain the values of parametric variables $u$ and $v$. By doing so, the values of the parametric variables $u$ and $v$ for all the points to be used for shape reconstruction are obtained. That is, for each point $\boldsymbol{X}_{n}$, we obtain its parametric values $u_{n}$ and $v_{n}$.

If $N$ points $\boldsymbol{X}_{n}(n=1,2,3, \cdots, N)$ are to be used to reconstruct a PDE surface patch $\mathbf{X}(u, v)$, we can calculate the squared sum of the errors between the known points $\boldsymbol{X}_{n}$ $(n=1,2,3, \cdots, N)$ and the unknown points $\mathbf{X}\left(u_{n}, v_{n}\right)$ with the following equation

$$
\begin{aligned}
\boldsymbol{E} & =\sum_{n=1}^{N}\left[\mathbf{X}\left(u_{n}, v_{n}\right)-\boldsymbol{X}_{n}\right]^{2} \\
& =\sum_{n=1}^{N}\left[\sum_{j=1}^{16} \boldsymbol{d}_{j} \boldsymbol{f}_{j}\left(u_{n}, v_{n}\right)-\boldsymbol{X}_{n}\right]^{2}
\end{aligned}
$$

The least squares are used to minimize the error $\boldsymbol{E}$ and find the 16 vector-valued unknowns with the equation below

$$
\begin{aligned}
& \frac{\partial \boldsymbol{E}}{\partial \boldsymbol{d}_{k}}=0 \\
& (k=1,2,3, \cdots, 16)
\end{aligned}
$$

Substituting Eq. (6) into Eq. (7), we obtain the following equations which can be used to determine the 16 vector-valued unknowns $\boldsymbol{d}_{j}(j=1,2,3, \cdots, 16)$

$$
\begin{gathered}
\sum_{j=1}^{16} \boldsymbol{d}_{j} \sum_{n=1}^{N} \boldsymbol{f}_{j}\left(u_{n}, v_{n}\right) \boldsymbol{f}_{k}\left(u_{n}, v_{n}\right)=\sum_{n=1}^{N} \boldsymbol{X}_{n} \boldsymbol{f}_{k}\left(u_{n}, v_{n}\right) \\
(k=1,2,3, \cdots, 16)
\end{gathered}
$$

Equation (8) involves 16 equations which can be used to determine the 16 vectorvalued unknowns $\boldsymbol{d}_{j}(j=1,2,3, \cdots, 16)$. 
$\boldsymbol{f}_{j}\left(u_{n}, v_{n}\right)$ and $\boldsymbol{f}_{k}\left(u_{n}, v_{n}\right)$ in Eq. (8) involves the constants $\boldsymbol{q}_{2}$ and $\boldsymbol{q}_{4}$, which are determined by $\boldsymbol{c}_{0}, \boldsymbol{a}_{1}$ and $\boldsymbol{a}_{2}$, respectively. $\boldsymbol{a}_{1}$ and $\boldsymbol{a}_{2}$ are vector-valued shape control parameters. They can be optimized to obtain the optimal PDE surface patch which best fits to the points $\boldsymbol{X}_{n}(n=1,2,3, \cdots, N)$.

However, if we take $\boldsymbol{q}_{2}$ and $\boldsymbol{q}_{4}$ as design variables, Eq. (8) becomes nonlinear. Solving Eq. (8) is to solve 16 nonlinear equations which makes the determination of the 16 vector-valued unknowns $\boldsymbol{d}_{j}(j=1,2,3, \cdots, 16)$ more difficult. Instead of optimizing $\boldsymbol{q}_{2}$ and $\boldsymbol{q}_{4}$ to find their optimal values, we set $\boldsymbol{q}_{2}$ and $\boldsymbol{q}_{4}$ to different values, solve the 16 linear algebra equations of Eq. (8), and find that $\boldsymbol{q}_{2}=0.1$ and $\boldsymbol{q}_{4}=0.1$ give good results.

In what follows, we use Eq. (3) to reconstruct a 3D PDE surface from different points, and compare the surface defined by the original points and the reconstructed PDE surface. In order to quantify the differences between the two surfaces, we calculate the maximum error and the average error between the two surfaces with the following equations

$$
\begin{gathered}
\operatorname{Err} M=\max \left\{\left|\boldsymbol{X}_{1}-\mathbf{X}\left(u_{1}, v_{1}\right)\right| \quad\left|\boldsymbol{X}_{2}-\mathbf{X}\left(u_{2}, v_{2}\right)\right| \quad \cdots \quad\left|\boldsymbol{X}_{N}-\mathbf{X}\left(u_{N}, v_{N}\right)\right|\right\} \\
\operatorname{Err} A=\frac{1}{N} \sum_{n=1}^{N}\left|\boldsymbol{X}_{n}-\mathbf{X}\left(u_{n}, v_{n}\right)\right|
\end{gathered}
$$

where $\operatorname{Err} M$ indicates the maximum error between the two surfaces, $\operatorname{Err} A$ indicates the average error between the two surfaces, $|\cdot|$ indicates the distance between the correspondent points of the two surfaces.

Firstly, we consider reconstructing a surface from 16 points give in Table 1 . Since a PDE surface patch (3) involves 16 unknowns, the PDE surface patch should pass through the 16 points if interpolation operation is used to determine the 16 unknowns. In this paper, the fitting operation defined by Eq. (8), not interpolation operation, is used to determine the 16 unknowns. Although the interpolation operation is not used, it is expected that the fitting operation should give high accuracy if not passing the 16 points.

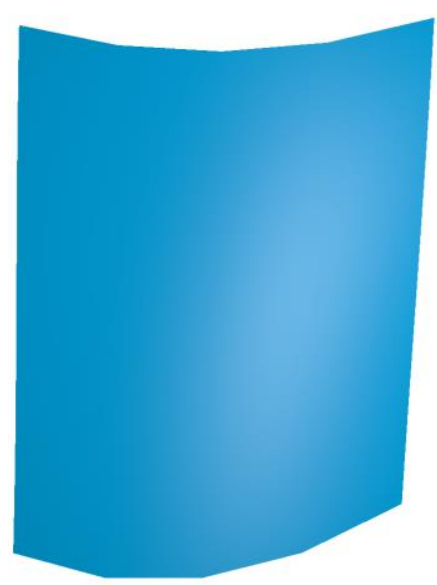

(a)

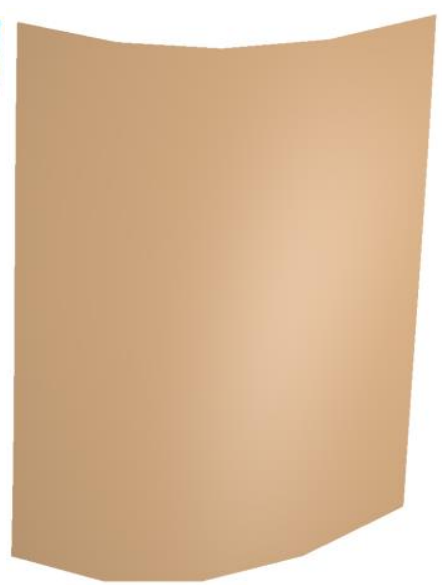

(b)

Fig. 1. Surface defined by 16 original points and the reconstructed PDE Surface. 
Table 1. 16 points used to define the surface in Fig. 1

\begin{tabular}{|c|c|c|c|}
\hline$(x, y, z)$ & $(x, y, z)$ & $(x, y, z)$ & $(x, y, z)$ \\
\hline$(0.05,-0.07,-1.12)$ & $(0.07,-0.07,-1.11)$ & $(0.10,-0.07,-1.11)$ & $(0.13,-0.07,-1.12)$ \\
\hline$(0.04,-0.10,-1.10)$ & $(0.07,-0.10,-1.09)$ & $(0.10,-0.10,-1.10)$ & $(0.13,-0.10,-1.10)$ \\
\hline$(0.04,-0.13,-1.08)$ & $(0.07,-0.13,-1.06)$ & $(0.10,-0.13,-1.07)$ & $(0.13,-0.13,-1.09)$ \\
\hline$(0.04,-0.16,-1.05)$ & $(0.07,-0.16,-1.04)$ & $(0.10,-0.16,-1.04)$ & $(0.13,-0.16,-1.06)$ \\
\hline
\end{tabular}

Setting $N=16$ in Eq. (8) and solving the 16 linear algebra equations, we obtain the 16 vector-valued unknowns. With the original 16 points, we create the surface depicted in Fig. 1(a). Substituting the obtained 16 vector-valued unknowns back into Eq. (3), we use Eq. (3) to create the PDE surface patch shown in Fig. 1(b). The maximum error and the average error between the two surfaces are given in Table 2.

Comparing the surface in Fig. 1(a) and the surface in Fig. 1(b), we could not find any differences, indicating the reconstructed PDE surface is the same as the original surface defined by the 16 points. This observation is also supported by the maximum error and average error given in Table 2 . The maximum error between the two surfaces $5.52 \times 10^{-5}$ and the average error between the two surfaces is $2.31 \times 10^{-5}$. Both errors are very small, which indicating high accuracy of the fitting operation.

Table 2. Maximum errors and average errors between the two surfaces

\begin{tabular}{|c|l|l|l|l|l|l|}
\hline$N$ & 16 & 25 & 36 & 49 & 64 & 81 \\
\hline ErrM & $5.52 \times 10^{-5}$ & $1.56 \times 10^{-3}$ & $2.96 \times 10^{-3}$ & $9.68 \times 10^{-3}$ & $1.40 \times 10^{-2}$ & $2.02 \times 10^{-2}$ \\
\hline ErrA & $2.31 \times 10^{-5}$ & $4.47 \times 10^{-4}$ & $1.33 \times 10^{-3}$ & $3.80 \times 10^{-3}$ & $5.76 \times 10^{-3}$ & $8.16 \times 10^{-3}$ \\
\hline
\end{tabular}

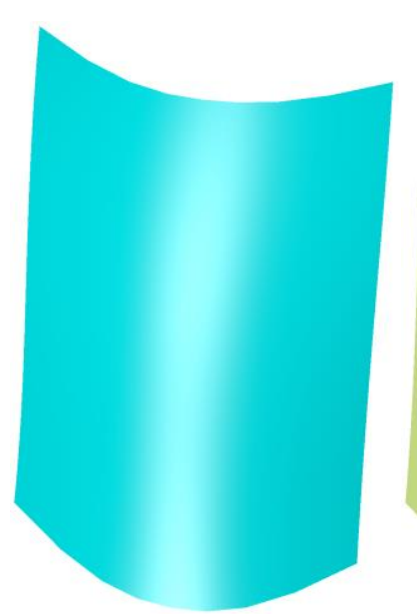

(a)

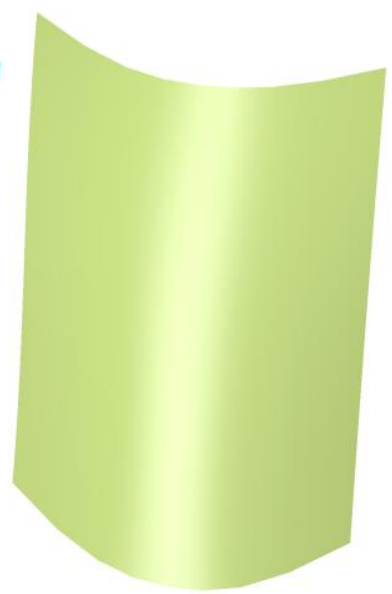

(b)

Fig. 2. Surface defined by 25 original points and the reconstructed PDE surface.

Secondly, we consider reconstructing a surface from 25 points consisting of those in Table 1 and Table 3. Setting $N=25$ in Eq. (8) and solving the 16 linear algebra equations, we obtain the 16 vector-valued unknowns. With the original 25 points, we create the surface depicted in Fig. 2(a). Substituting the obtained 16 vector-valued unknowns 
back into Eq. (3), we use Eq. (3) to create the PDE surface shown in Fig. 2(b). The maximum error and the average error between the two surfaces are given in Table 2 .

Table 3. Points used with those in Table 1 to define the surface in Fig. 2

\begin{tabular}{|c|c|c|c|}
\hline$(x, y, z)$ & $(x, y, z)$ & $(x, y, z)$ & $(x, y, z)$ \\
\hline$(0.02,-0.07,-1.16)$ & $(0.02,-0.10,-1.16)$ & $(0.07,-0.19,-1.01)$ & $(0.10,-0.19,-1.01)$ \\
\hline$(0.01,-0.19,-1.05)$ & $(0.04,-0.19,-1.02)$ & $(0.10,-0.10,-1.10)$ & $(0.13,-0.10,-1.10)$ \\
\hline$(0.13,-0.19,-1.03)$ & & & \\
\hline
\end{tabular}

Comparing the surface in Fig. 2(a) and the surface in Fig. 2(c), we still could not find any differences, indicating the reconstructed PDE surface is very similar to the original surface defined by the 25 points. As indicated in Table 2, the maximum error between the two surfaces is $1.56 \times 10^{-3}$ and the average error between the two surfaces is $4.47 \times 10^{-4}$, indicating small errors between the original surface and the reconstructed PDE surface.

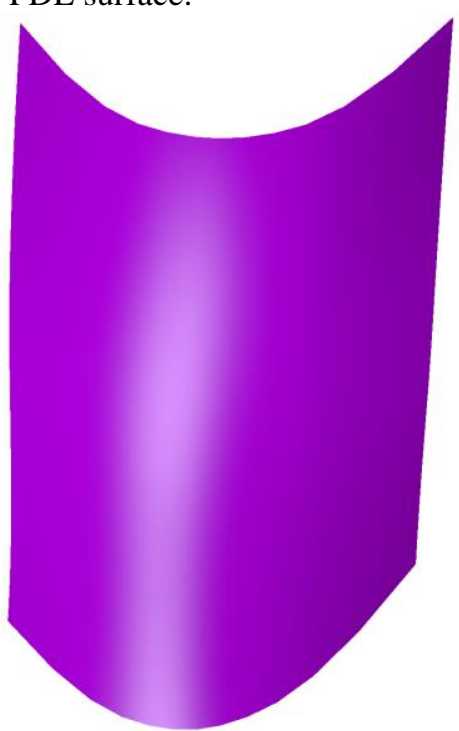

(a)

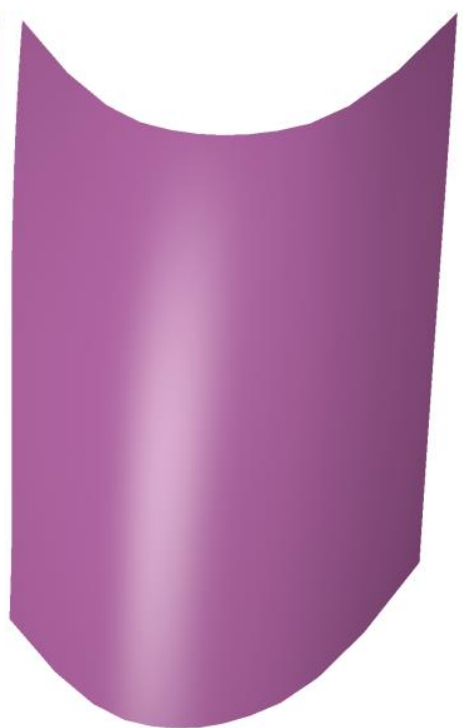

(b)

Fig. 3. Surface defined by 36 original points and the reconstructed PDE surface.

Thirdly, we consider reconstructing a surface from 36 points consisting of those in Table 1, Table 3, and Table 4. Setting $N=36$ in Eq. (8) and solving the 16 linear algebra equations, we obtain the 16 vector-valued unknowns. With the original 36 points, we create the surface depicted in Fig. 3(a). Substituting the obtained 16 vector-valued unknowns back into Eq. (3), we use Eq. (3) to create the PDE surface shown in Fig. 3(b). The maximum error and the average error between the two surfaces are given in Table 2.

Comparing the surface in Fig. 3(a) and the surface in Fig. 3(b), no obvious differences could be found, indicating the reconstructed PDE surface gives a good approxi- 
mation to the original surface defined by the 36 points. As shown in Table 2, the maximum error between the two surfaces is $2.96 \times 10^{-3}$ and the average error between the two surfaces is $1.33 \times 10^{-3}$. Although design variables have been reduced by more than a half, i. e., from $36 \times 3=108$ to $16 \times 3=48$, the maximum error and average error are small.

Table 4. Points used with those in Tables 1 and 3 to define the surface in Fig. 3

\begin{tabular}{|c|c|c|c|}
\hline$(x, y, z)$ & $(x, y, z)$ & $(x, y, z)$ & $(x, y, z)$ \\
\hline$(0.02,-0.04,-1.20)$ & $(0.05,-0.04,-1.15)$ & $(0.07,-0.04,-1.14)$ & $(0.10,-0.04,-1.14)$ \\
\hline$(0.13,-0.04,-1.15)$ & $(0.17,-0.03,-1.20)$ & $(0.17,-0.07,-1.17)$ & $(0.16,-0.10,-1.14)$ \\
\hline$(0.16,-0.13,-1.12)$ & $(0.16,-0.16,-1.10)$ & $(0.16,-0.19,-1.07)$ & \\
\hline
\end{tabular}

Fourthly, we consider reconstructing a surface from 49 points. Setting $N=49$ in Eq. (8) and solving the 16 linear algebra equations, we obtain the 16 vector-valued unknowns. With the original 49 points, we create the surface depicted in Fig. 4(a). Substituting the obtained 16 vector-valued unknowns back into Eq. (3), we use Eq. (3) to create the PDE surface patch shown in Fig. 4(b). The maximum error and the average error between the two surfaces are given in Table 2 .

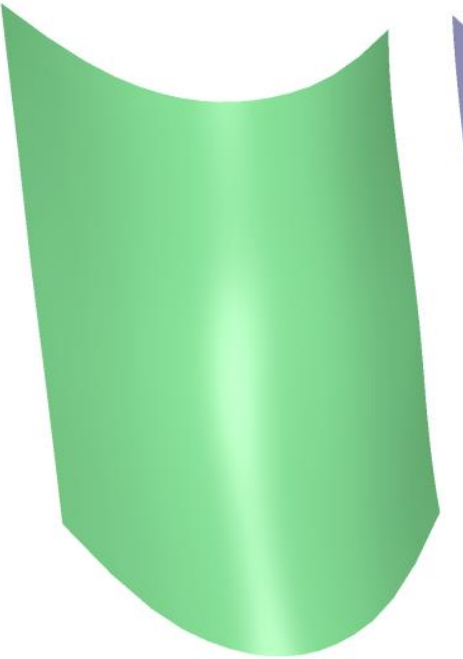

(a)

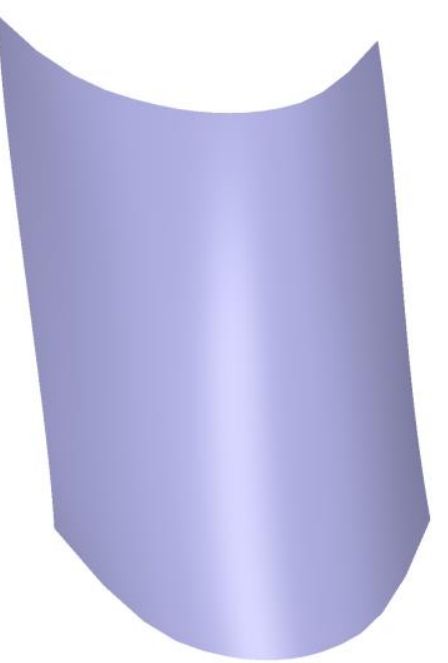

(b)

Fig. 4. Surface defined by 49 original points and the reconstructed PDE surface.

The surface in Fig. 4(a) looks like the surface in Fig. 4(b). As indicted in Table 2, the maximum error between the two surfaces is $9.68 \times 10^{-3}$ and the average error between the two surfaces is $3.80 \times 10^{-3}$. For this case, the design variables are reduced by two thirds, i. e., from $49 \times 3=147$ to $16 \times 3=48$, the maximum error and average error are still small.

Fifthly, we consider reconstructing a surface from 64 points. Setting $N=64$ in Eq. (8) and solving the 16 linear algebra equations, we obtain the 16 vector-valued un- 
knowns. With the original 64 points, we create the surface depicted in Fig. 5(a). Substituting the obtained 16 vector-valued unknowns back into Eq. (3), we use Eq. (3) to create the PDE surface shown in Fig. 5(b). The maximum error and the average error between the two surfaces are given in Table 2 .

The surface in Fig. 5(a) still looks like the surface in Fig. 5(b). The maximum error between the two surfaces is $1.40 \times 10^{-2}$ and the average error between the two surfaces is $5.76 \times 10^{-3}$. For this case, the design variables are reduced by three fourths, $i$. e., from $64 \times 3=192$ to $16 \times 3=48$, the maximum error and average error are not big.

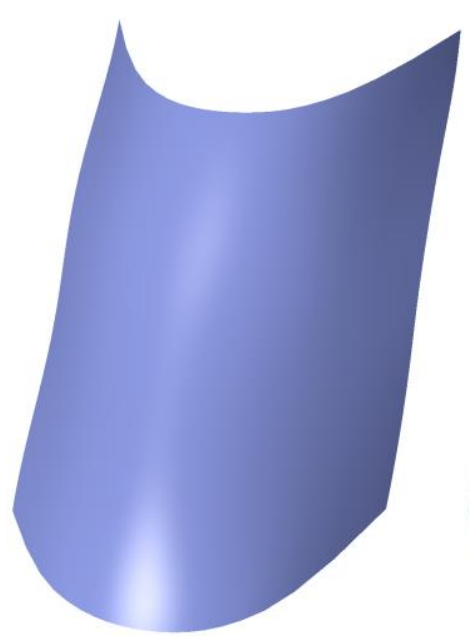

(a)

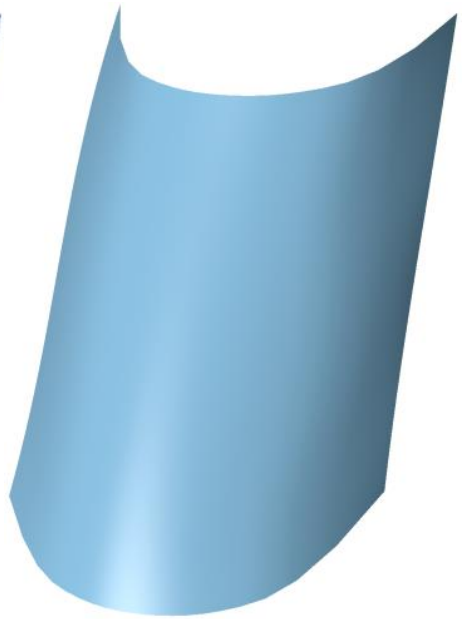

(b)

Fig. 5. Surface defined by 64 original points and the reconstructed PDE surface.

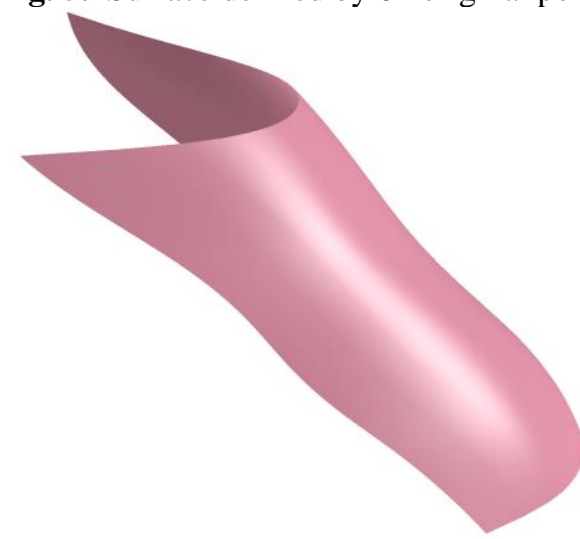

(a)

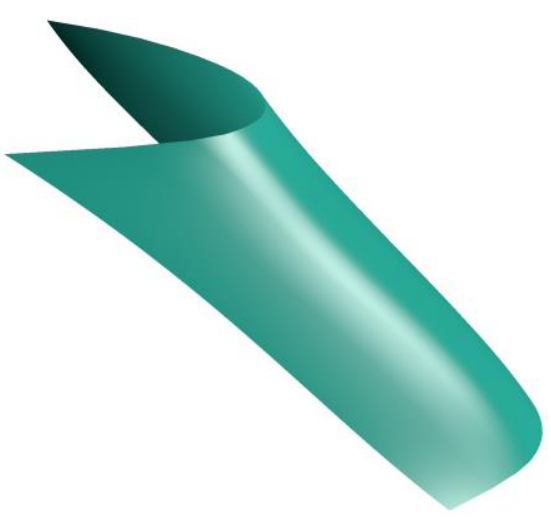

(b)

Fig. 6. Surface defined by 81 original points and the reconstructed PDE surface.

Finally, we consider reconstructing a surface from 81 points. Setting $N=81$ in Eq. (8) and solving the 16 linear algebra equations, we obtain the 16 vector-valued unknowns. With the original 81 points, we create the surface depicted in Fig. 6(a). Substituting the obtained 16 vector-valued unknowns back into Eq. (3), we use Eq. (3) to 
create the PDE surface shown in Fig. 6(b). The maximum error and the average error between the two surfaces are given in Table 2 .

Since the design variables have been reduced from $81 \times 3=243$ to $16 \times 3=48$, i. e., reduced by four fifths, some differences can be observed from the two surfaces depicted in Fig. 6(a) and 6(b). As shown in Table 2, the maximum error between the two surfaces becomes $2.02 \times 10^{-2}$ and the average error between the two surfaces becomes $8.16 \times 10^{-3}$, which are not big.

\section{Conclusions}

In this paper, we have proposed a new method for shape reconstruction from point clouds. It is based on accurate closed form solutions to a vector-valued fourth-order partial differential equation. Due to the nature of explicit analytical expressions of the reconstructed surface, the proposed method is very efficient in reconstructing 3D surfaces from point clouds. The error analysis given in this paper demonstrates good approximation of the reconstructed surface from the proposed PDE-based shape reconstruction method to point clouds.

There are a number of directions that can be investigated in the following work. First, the particular solution of the nonhomogeneous equation of Eq. (2) should be developed to make the proposed PDE-based shape reconstruction more powerful. Second, how to use the obtained solutions of the proposed vector-valued fourth-order partial differential equation to reconstruct unorganized point clouds with noise and missing data should be examined. In this paper, only shape reconstruction by using one single PDE surface patch was discussed. For complicated 3D models, a single PDE surface patch is unable to reconstruct complicated 3D models. How to use multiple PDE surface patches to reconstruct complicated $3 \mathrm{D}$ models from point clouds should be considered. The vector-valued shape control parameters involved in the PDE (2) affects the shape of reconstructed surfaces. How to identify an efficient and effective optimization method and combine it with a suitable method of solving the nonlinear equations (8) to find optimal values of the two parameters $\boldsymbol{q}_{2}$ and $\boldsymbol{q}_{4}$, and obtain the 16 vector-valued unknowns to maximum the potential of the proposed PDE-based shape reconstruction should also be explored. Another interesting direction is using the obtained closed form solutions to accurately satisfy all the constraints on four boundaries of a PDE patch. To do this, Eq. (3) can be extended into a series $\mathbf{X}(u, v)=\sum_{m}^{M} \sum_{j=1}^{16} \boldsymbol{d}_{m j} \boldsymbol{f}_{m j}(u, v)$ with $\boldsymbol{q}_{2}$ and $\boldsymbol{q}_{4}$ being replaced by $\boldsymbol{q}_{2 m}$ and $\boldsymbol{q}_{4 m}$. We will investigate this in our following work.

\section{Acknowledgements}

This research is supported by the PDE-GIR project which has received funding from the European Union Horizon 2020 research and innovation programme under the Marie Skodowska-Curie grant agreement No 778035. 


\section{References}

1. Berger, M., Tagliasacchi, A., Seversky, L.M., Alliez, P., Levine, J.A., Sharf, A., Silva, C.T.: State of the art in surface reconstruction from point clouds. Eurographics 2014 - State of the Art Reports, pp.161-185, (2014).

2. Berger, M., Tagliasacchi, A., Seversky, L.M., Alliez, P., Guennebaud, G., Levine, J.A., Sharf, A., Silva, C.T.: A survey of surface reconstruction from point clouds. Computer Graphics Forum 36(1), 301-329 (2017).

3. Othman, M.N.M., Yusoff, Y., Haron, H., You, L.H.: An overview of surface reconstruction using partial differential equation (PDE). IOP Conf. Series: Materials Science and Engineering 551, 1-5 (2019).

4. Boissonnant, J.D.: Geometric structures for three-dimensional shape reconstruction. ACM Transactions on Graphics 3(4), 266-289 (1984).

5. Hoppe, H., DeRose, T., Duchamp, T., McDonald, J., Stuetzle, W.: Surface reconstruction from unorganized points. In Proceedings of SIGGRAPH 1992, pp. $71-78$ (1992).

6. Oblonšek, Č., Guid, N.: A fast surface-based procedure for object reconstruction from 3D scattered points. Computer Vision and Image Understanding 69(2), 185-195 (1998).

7. Bernardini, F., Mittelman, J., Rushmeier, H., Silva, C., Taubin, G.: The ball-pivoting algorithm for surface reconstruction. IEEE Transactions on Visualization and Computer Graphics 5(4), 349-359 (1999).

8. Gopi, M., Krisnan, S., Silva, C.: Surface reconstruction based on lower dimensional localized Delaunay triangulation. Computer Graphics Forum 19(3), 467-478 (2000).

9. Lee, A., Moreton, H., Hoppe, H.: 2000: Displaced subdivision surfaces. Proceedings of SIGGRAPH 2000, pp. 85-94 (2000).

10. Jeong, W.K., Kim, C.H.: Direct reconstruction of displaced subdivision surface from unorganized points. Graphical Models 64(2), 78-93 (2002).

11. Nan, L., Wonka, P.: PolyFit: Polygonal surface reconstruction from point clouds. 2017 IEEE International Conference on Computer Vision (ICCV), Venice, pp. 2372-2380 (2017).

12. Duan, Y., Yang, L., Qin, H., Samaras, D.: Shape reconstruction from 3D and 2D data using PDE-based deformable surfaces. Lecture Notes in Computer Science 3023, 238-251 (2004).

13. Linz, C., Goldlücke, B., Magnor, M.: A point-based approach to PDE-based surface reconstruction. Lecture Notes in Computer Science 4174, 729-738 (2006).

14. Franchini, E., Morigi, S., Sgallari, F.: Implicit shape reconstruction of unorganized points using PDE-based deformable 3D manifolds. Numerical Mathematics: Theory, Methods and Applications 3(4), 405-430 (2010).

15. Pana, R., Skala, V.: Continuous global optimization in surface reconstruction from an oriented point cloud. Computer-Aided Design 43, 896-901 (2011).

16. Liu, W., Cheung, Y., Sabouri, P., Arai, T.J., Sawant, A., Ruan, D.: A continuous surface reconstruction method on point cloud captured from a 3D surface photogrammetry system. Medical Physics 42(11), 6564-6571 (2015).

17. He, Y., Huska, M., Kang, S.H., Liu, H.: Fast algorithms for surface reconstruction from point cloud, https://arxiv.org/abs/1907.01142, pp. 1-16 (2019).

18. Li, J., Hero, A.O.: A fast spectral method for active 3D shape reconstruction. Journal of Mathematical Imaging and Vision 20, $73-87$ (2004).

19. Ugail, H., Kirmani, S.: Method of surface reconstruction using partial differential equations. Proceedings of the 10th WSEAS International Conference on COMPUTERS, Vouliagmeni, Athens, Greece, July 13-15, pp. pp51-56 (2006).

20. Elyan, E., Ugail, H.: Reconstruction of 3D human facial images using partial differential equations. Journal of Computers 2 (8): 1-8 (2007). 
21. Rodrigues, M., Osman, A., Robinson, A.: Partial differential equations for 3D data compression and reconstruction. Advances in Dynamical Systems and Applications 8(2): 303-315 (2013).

22. Sheng, B., Zhao, F., Yin, X., Zhang, C., Wang, H., Huang, P.: A lightweight surface reconstruction method for online 3D scanning point cloud data oriented toward 3D printing. Mathematical Problems in Engineering 2018, Article ID 4673849: 1-16 (2018).

23. Iglesias, A., Echevarría, G., Gálvez, A.: Functional networks for B-spline surface reconstruction. Future Generation Computer Systems 20(8), 1337-1353 (2004).

24. Gálvez, A., Iglesias, A.: Iterative two-step genetic-algorithm-based method for efficient polynomial B-spline surface reconstruction. Information Sciences 182(1), 56-76 (2012).

25. Gálvez, A., Iglesias, A.: Particle swarm optimization for non-uniform rational B-spline surface reconstruction from clouds of 3D data points. Information Sciences 192(1), 174-192 (2012). 